Distribution Category: Atomic, Molecular, and Chemical Physics (UC-411)

\author{
ARGONNE NATIONAL LABORATORY \\ 9700 South Cass Avenue \\ Argonne, Illinois 60439
}

ANT/APS/TB--2

DE93 001338

ANL/APS/TB-2

\title{
Use of a Mirror as the First Optical Component for an Undulator Beamline at the APS
}

\author{
by W. Yun, A. Khounsary, B. Lai, and E. Gluskin \\ Experimental Facilities Division \\ Advanced Photon Source
}

September 1992

work sponsored by

U.S. DEPARTMENT OF ENERGY

Office of Energy Research 


\title{
Use of a Mirror as the First Optical Component for an Undulator Beamline at the APS
}

\author{
W. Yun, A Khounsary, B. Lai, and E. Gluskin
}

\section{Introduction}

In the design of Sector II of the Synchrotron Radiation Instrumentation (SRI) CAT, an x-ray mirror with multiple coatings is chosen as the first optical component of the undulator beamline. Two significant advantages of using the mirror are: (1) a significant reduction in the peak radiation heat flux and total power on the downstream monochromator, and (2) availability of the wide-bandpass undulator spectrum between $0-30 \mathrm{keV}$ to experimental stations with substantially reduced radiation shielding requirements. The second advantage also allows us to place the monochromator outside the first optics enclosure (FOE) at a large distance from the source to further reduce the peak heat flux on the monochromator. The combined effect is that the inclined crystal monochromator ${ }^{1}$ may not be necessary, and a multilayer monochromator can be used because the expected heat fluxes are less than the value that has been demonstrated for those monochromators. 2,3

In this technical note, unless otherwise stated, the energy of the stored positron beam is $7 \mathrm{GeV}$ and the current is $100 \mathrm{~mA}$.

\section{Thermal Considerations}

The mirror described here is vertically deflecting to minimize the mirror size and is located 30.5 meters from the source. The grazing incidence angle is $0.15^{\circ}$. The mirror length is $90 \mathrm{~cm}$, and the mirror intercepts 77 $\mu \mathrm{rad}$ of incident beam in the vertical direction. This incidence angle of $0.15^{\circ}$ is selected to obtain high reflectivities for x-ray energies up to $30 \mathrm{keV}$ (see Fig. 1) and to produce an offset of about $25 \mathrm{~mm}$ for the reflected beam at the exit wall of the FOE. This is necessary to limit Bremsstrahlung shielding beyond the FOE. The peak heat flux incident on the mirror surface is 0.35 $\mathrm{W} / \mathrm{mm}^{2}$. At this power density, thermally induced slope errors less than 1 arcsec may be obtainable because a slope error of about 2 arcsec was obtained for a power density of about $2 \mathrm{~W} / \mathrm{mm}^{2}$, but at a lower total power than our mirror will experience. 4

The mirror has three coating materials ( $\mathrm{Al}, \mathrm{Rh}$, and $\mathrm{Pt}$ ) that are deposited in three parallel strips on the same substrate. Fig. 1 shows the calculated $\mathrm{x}$-ray reflectivity as a function of $\mathrm{x}$-ray energy for a $0.15^{\circ}$ grazing incidence angle. The three coating materials are necessary to reduce the peak heat flux on the monochromator to less than $4 \mathrm{~W} / \mathrm{mm}^{2}$ for the $4.2-30 \mathrm{keV}$ energy 
range of the Undulator A spectrum. For a heat flux of $4 \mathrm{~W} / \mathrm{mm}^{2}$, a conventional double-crystal monochromator (using gallium cooling) that has been developed at APS can be used. 2

The peak power density of the raw Undulator A spectrum as a function of the undulator gap size is shown in Fig. 2. Also shown in the figure are the power densities of the undulator spectrum integrated over the three energy bands that are indicated in the figure. It is clear from the figure that a substantial reduction in the peak power density can be obtained by filtering out the high energy part of the raw undulator spectrum. Because the x-ray reflectivity of a mirror is small for x-rays of energy greater than the cut-off energy at a given incidence angle, the power densities calculated for the three energy-integration bands in Fig. 2 can be approximated as the power densities of the Undulator A spectrum reflected by three mirrors with their cut-off energies being equal to 10,20 , and $30 \mathrm{keV}$, respectively. The cut-off energy is defined here as the energy at which $x$-ray reflectivity drops to about 0.5 . Note that a reduction of power density of about a factor 10 is obtained at the closed gap of $11.5 \mathrm{~mm}$ when a mirror with a cut-off energy of $10 \mathrm{keV}$ is used.

In addition to the power density reduction, the total power of Undulator A reflected by a mirror is also reduced. Fig. 3 shows the total power of the raw Undulator A spectrum as the function of the undulator gap size. Also shown in the figure are the total powers of the spectrum integrated over the four energy-integration bands that are indicated. The reduction of the total power reflected by a mirror reduces the total power load on the monochromator and other downstream optical components, and, thus, the design of these components can be more flexible.

The double crystal monochromator is located at 56 meters from the center of Undulator A. The normal incidence heat fluxes at the monochromator for the raw Undulator A spectrum and for the three energy-integration bands are also plotted in Fig. 2. In practice, the beam is incident on the monochromator at an angle less than 90 degrees because the monochromator is tuned to diffract either the first or the third harmonic of the undulator radiation. Figs. $4 \& 5$ show the heat flux on a $\mathrm{Si}(111)$ monochromator surface when it is tuned to diffract the first and the third harmonic radiation of the Undulator A spectrum, respectively. For the 4.2 $14 \mathrm{keV}$ energy tuning range of the Undulator A first harmonic radiation, the heat flux on the monochromator is less than $4 \mathrm{~W} / \mathrm{mm}^{2}$ if an Al mirror is used for the $4.2-10 \mathrm{keV}$ energy range and either a $\mathrm{Rh}$ or $\mathrm{Pt}$ mirror is used for the $10-14 \mathrm{keV}$ energy range (Fig. 4). For the tuning range of third harmonic radiation, $12.6-42 \mathrm{keV}$, the peak heat fluxes on the monochromator for all the three energy-integration bands are less than 4 $\mathrm{W} / \mathrm{mm}^{2}$ (Fig. 5). Because the cut-off energy of the $\mathrm{Al}$ mirror is about $12 \mathrm{keV}$ at the 0.15 degree of grazing incidence angle, only the $\mathrm{Rh}$ or $\mathrm{Pt}$ mirror will be used for harmonic radiation of energy larger than $12 \mathrm{keV}$. Note from Fig. 2, however, that since the normal incidence heat flux at the monochromator can be as high as about $18 \mathrm{~W} / \mathrm{mm}^{2}$ from a $\mathrm{Rh}$ or $\mathrm{Pt}$ mirror, 
it may be necessary to interlock both the mirror being selected and the monochromator angular position in order to protect the monochromator from possible damage by ex essively high heat flux.

The thermal heat flux on a multilayer monochromator can also be estimated based on the results obtained above. Currently the smallest dspacing obtainable in a multilayer is about $20 \AA$, which is about 7 times that for $\mathrm{Si}(111)$. Therefore, the heat flux on the surface of a multilayer is at least 7 times smaller than that of a Si(111) crystal. Since the maximum heat flux on the surface of a Si(111) crystal can be kept to less than $4 \mathrm{~W} / \mathrm{mm}^{2}$ when the mirror is properly selected, the maximum heat flux on a multilayer monochromator should be kept to less than about $0.6 \mathrm{~W} / \mathrm{mm}^{2}$. The reduction of the power density results primarily from the smaller incidence angle for a multilayer monochromator than that for a $\mathrm{Si}$ crystal monochromator. This level of heat flux should not pose any real problem to most multilayers that are planned for use on our beamline because several types of multilayers have been shown to be able to resist heat fluxes as high as $7.5 \mathrm{~W} / \mathrm{mm}^{2} .3$ In addition, smaller heat fluxes are obtained for a multilayer monochromator with d-spacing larger than $20 \AA$ because a smaller grazing incidence angle has to be used.

In the table below, we list some key parameters relevant to tine thermal loading of the mirror and the monochromator. An Undulator A source is assumed. The maximum total power on the $\mathrm{Si}$ monochromator was obtained at the closed undulator gap and when a mirror with a $20-\mathrm{keV}$ cutoff energy is used. The $20-\mathrm{keV}$ cut-off energy was selected in order to use the $12.6-\mathrm{keV}$ third-order harmonic radiation at the closed gap.

\begin{tabular}{|l|l|l|}
\hline & Maximum Total Power & \multicolumn{1}{|c|}{ Peak HeatFlux } \\
\hline Entire Raw Spectrum & $3.8 \mathrm{~kW}$ & $\begin{array}{l}143 \mathrm{~W} / \mathrm{mm}^{2} @ 30.5 \mathrm{~m} \text { from } \\
\text { source and normal } \\
\text { incidence }\end{array}$ \\
\hline $\begin{array}{l}\text { Incidenton Mirror (after a } \\
77 \times 120 \mu \text { rad aperture) }\end{array}$ & $1 \mathrm{~kW}$ & $\begin{array}{l}0.38 \mathrm{~W} / \mathrm{mm}^{2} @ 0.15^{\circ} \text { grazing } \\
\text { incidenceangle }\end{array}$ \\
\hline $\begin{array}{l}\text { Incidenton Si } \\
\text { Monochromator @ 56m } \\
\text { from Source }\end{array}$ & $300 \mathrm{~W}$ & $\begin{array}{l}<\mathrm{W} / \mathrm{mm}^{2} \text { (se text for } \\
\text { conditions) }\end{array}$ \\
\hline
\end{tabular}




\section{Radiation Shielding Considerations}

The reduction of radiation shielding makes use of the low reflectivities of $\mathbf{x}$ rays of energies greater than the cut-off energy of a mirror (see Fig. 1). Fig. 6 shows both the raw Undulator A spectrum at closed gap of $11.5 \mathrm{~mm}$ and the spectrum being reflected from a $\mathrm{Pt}$ mirror at 0.15 degrees of grazing incidence angle. Also shown in the figure is the spectrum of the reflected beam after passing through a shielding slab consisting of 0.25 inch of lead and 0.125 inch of steel. The shielding structure specified here is similar to that of a vacuum tube that has been fabricated and tested at APS for shielding monochromatic beam.

To assess the radiation shielding requirements, two simple cases were considered for calculation of the radiation dose absorbed by human tissue. In the first case, the human tissue is assumed to be positioned behind the shielding slab that is in the direct path of Undulator A radiation after being reflected by the $\mathrm{Pt}$ mirror. In the second case, the shielding slab and the human tissue behind it were assumed to be exposed to isotropically scattered radiation of the Undulator A spectrum after being reflected by the $\mathrm{Pt}$. A point scatterer with $100 \%$ scattering efficiency located at the center of a 4-inch vacuum pipe was assumed. The distance between the human tissue and the scatter is assumed to be 2 inches. The doses for those two cases are about $2.8 \mathrm{~m} . \mathrm{rem} / \mathrm{s}$ and $0.6 \mathrm{mrem} / \mathrm{hr}$, respectively. While the calculated result in the first case indicates the back wall of a hutch needs to have a lead layer thicker than 0.25 inch, the calculated result in the second case seems to indicate that a 0.25 inch lead shield in the side wall of a vacuum transport cornponent may be enough to reduce the radiation dose to a safe level in our design. It is, however, necessary to perform a detailed calculation regarding the radiation shielding requirements. Nevertheless, the reduction in radiation shielding requirements is obtained by the use of a mirror, and this allows us to place the monochromator outside the FOE using standard APS vacuum transport components.

\section{Summary and Remarks}

X-ray mirrors can be used as a first optical component in an undulator beamline to reduce both the thermal loading on the monochromator in a way such that a conventional double-crystal or multilayer monochromator can be used and also to significantly reduce the radiation shielding requirements in a way that access to the wide-bandpass undulator radiation up to $30 \mathrm{keV}$ may be possible using the standard beamline vacuum transport components that are designed at APS.

In the preceding analysis, we have only considered the reduction of peak power density and the radiation shielding requirements for the case in which one of the three mirrors with different energy cut-offs is at a fixed incidence angle to the incident bean. Other possibilities, such as using two parallel mirrors at a fixed incidence angle or decreasing the incidence angle with the undulator gap size can also be considered. Significant 
reduction in the peak power density and the total power of the reflected beam and particularly in the radiation shielding requirements should be obtainable by the use of a two mirror system compared to the single mirror case considered here.

An example of the heat flux reduction using a pair of parallel mirrors tuned to have a cut-off energy to be 1.2 times the first harmonic radiation is illustrated in Fig. 7. This figure shows the peak heat fluxes at 56 meters from the source as a function of undulator gap for the spectrum reflected by the mirror pair as well as the gap-dependent heat fluxes for the entire Undulator A spectrum at normal incidence and at the surface of a $\mathrm{Si}(111)$ crystal tuned to diffract the first harmonic radiation. A significant reduction in the peak heat fluxes on a downstream monochromator is obtained if the mirror pair is used. In Fig. 7, the peak heat fluxes on the surface of a $\mathrm{Si}(111)$ crystal are also shown when both the mirror pair and the $\mathrm{Si}(111)$ crystal are tuned. Note that heat fluxes less than about 1.7 $\mathrm{W} / \mathrm{mm}^{2}$ on the crystal surface are obtained over the entire gap range.

In conclusion, we believe that an optimized thermal management scheme using a proper combination of mirrors and crystal geometry can be developed to handle most of the thermal loading problems with undulator radiation at APS. A combination of a mirror and an inclined monochromator ${ }^{1,2}$ may be able to handle even higher thermal loading problems that may arise with an increase of the energy and the current of the stored positron keam beyond the values calculated in this technical note and with increased undulator length. Expansion of the x-ray energy coverage beyoud $30 \mathrm{keV}$ may be obtained by having a strip of the mirror coated with a multilayer. At 0.15 degree of grazing incidence angle, $\mathbf{x}$-rays of energy up to $110 \mathrm{keV}$ can be reflected by a multilayer with a d-spacing of $20 \AA$ or longer.

\section{Acknowledgments:}

We would like to thank D. Shu, K. Randall, I. McNulty, and D. Legnini for useful discussions.

\section{References:}

1. A. Khounsary, Rev. Sci. Instrum. 63, 461 (1992).

2. R. K. Smither, W. Lee, A. Macrander, D. Mills, and C. S. Rogers, Rev. Sci. Instrum. 63, 1746 (1992); A. Macrander, W. Lee, R. K. Smither, D. Mills, and C. S. Rogers, and A. M. Khounsary, Nucl. Instrum. Methods, A319, 188 (1992).

3. E. Ziegler, G. Marot, A. K. Fraund, H. Kawata, L. E. Berman, and M. Iarocci, Rev. Sci. Instrum. 63, 496 (1992).

4. S. Mourikis, W. Jark, E. E. Koch, and V. Sail, Rev. Sci., Instr. 60, 1474 (1989). 


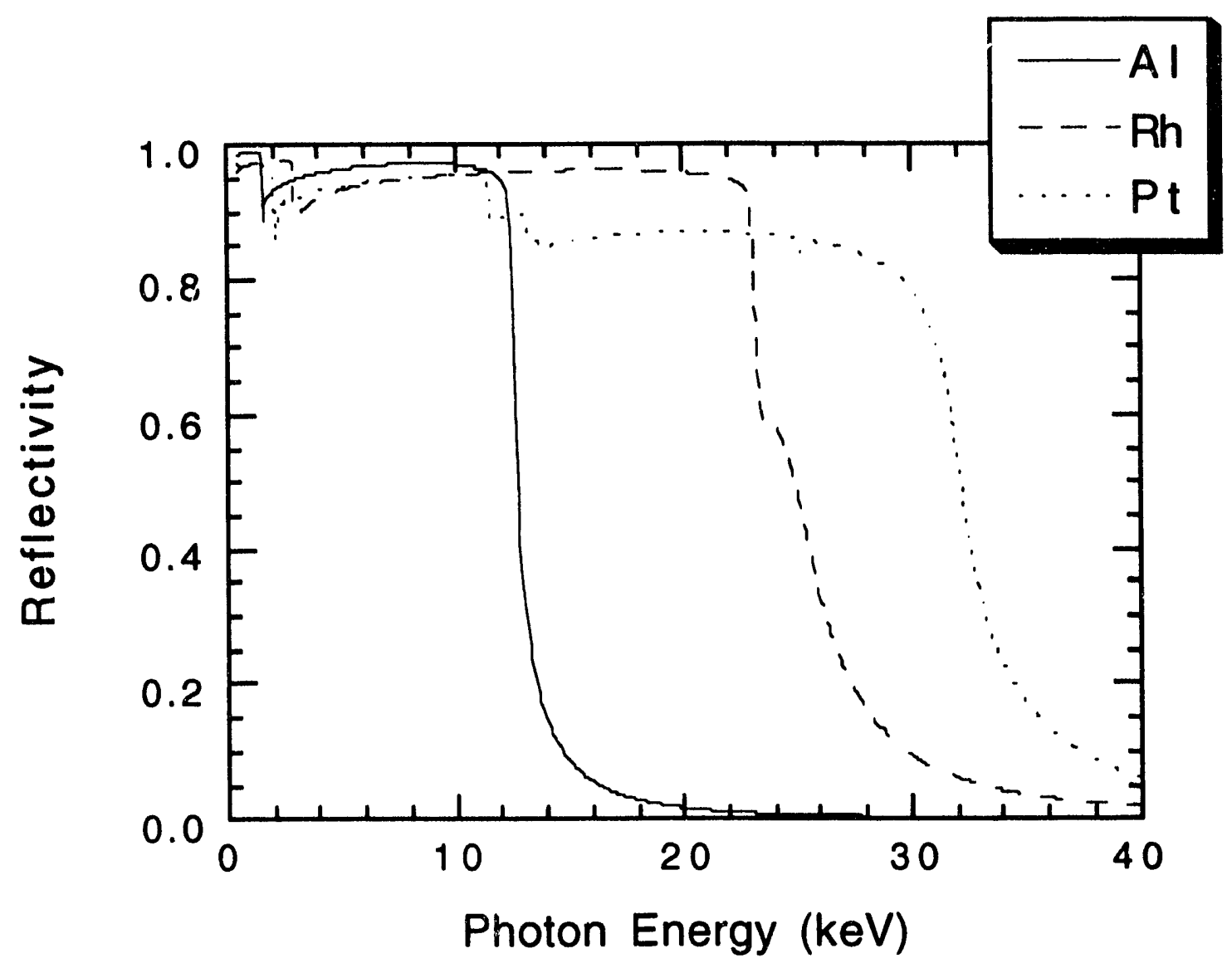

Fig. 1 X-ray reflectivity as a function of $\mathrm{x}$-ray energy for $\mathrm{Al}, \mathrm{Rh}$, and $\mathrm{Pt}$ mirrors at 0.15 degree of incidence angle. Note the cut-off energy for the $\mathrm{Pt}$ mirror is about $32 \mathrm{keV}$. 


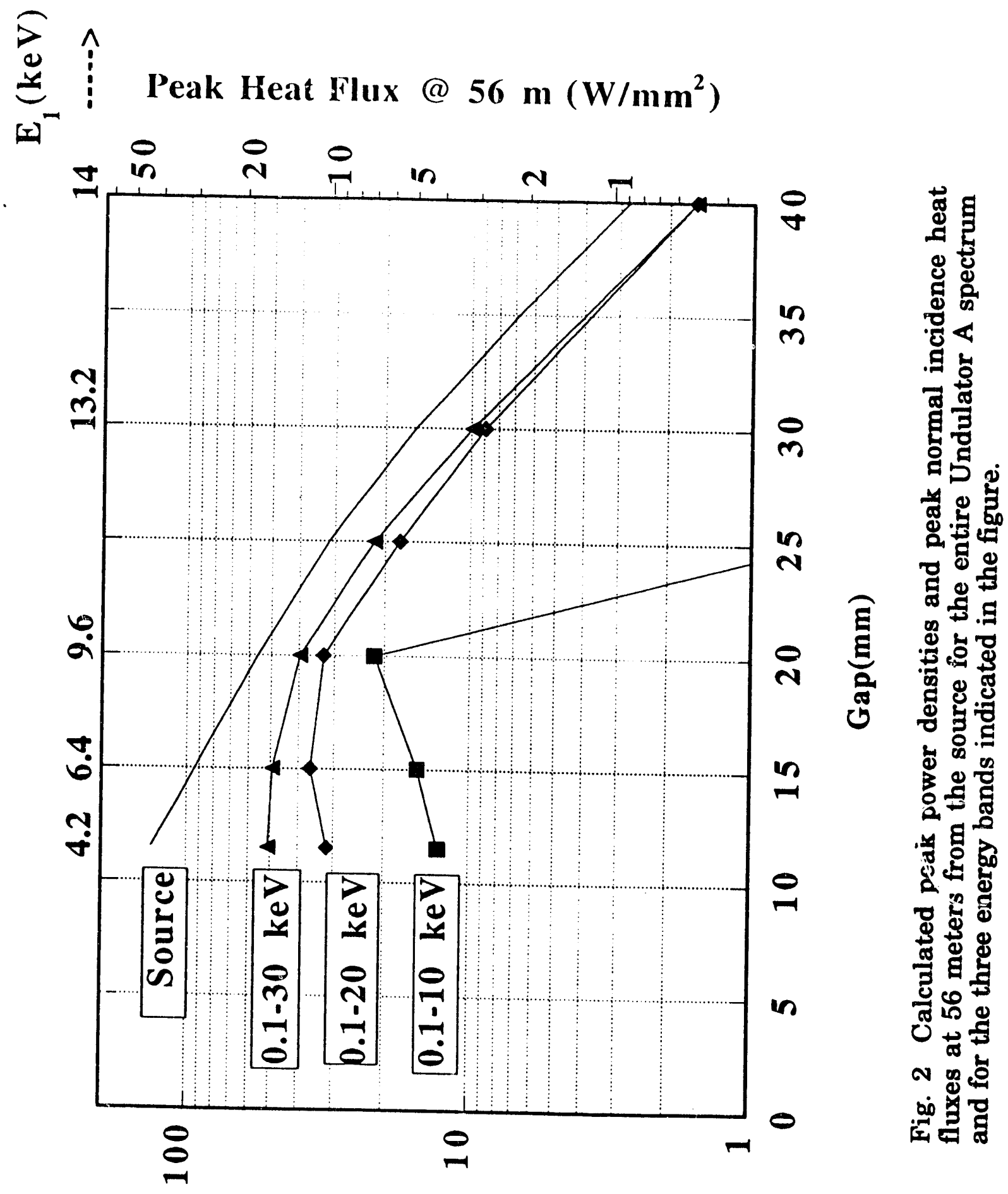

( 


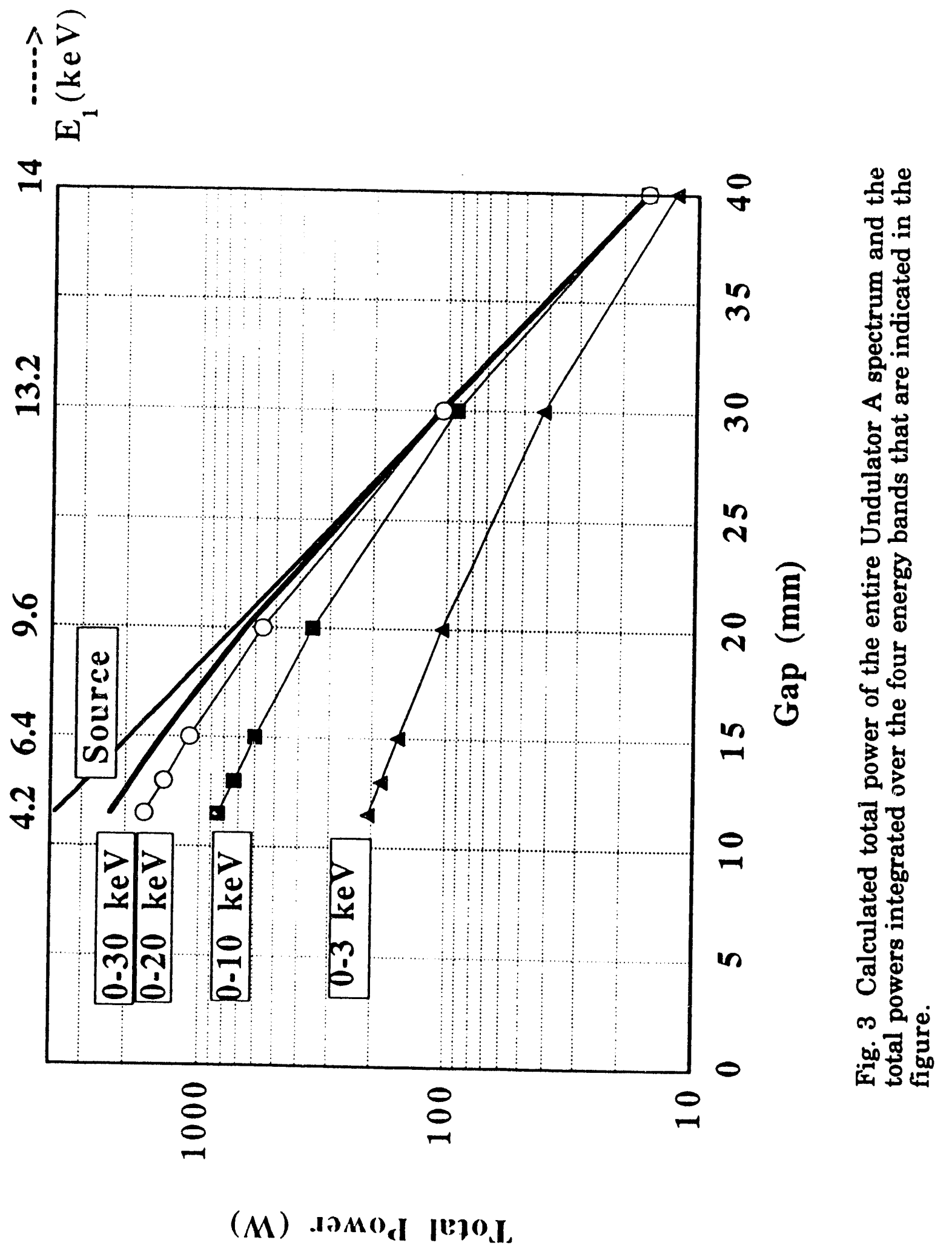




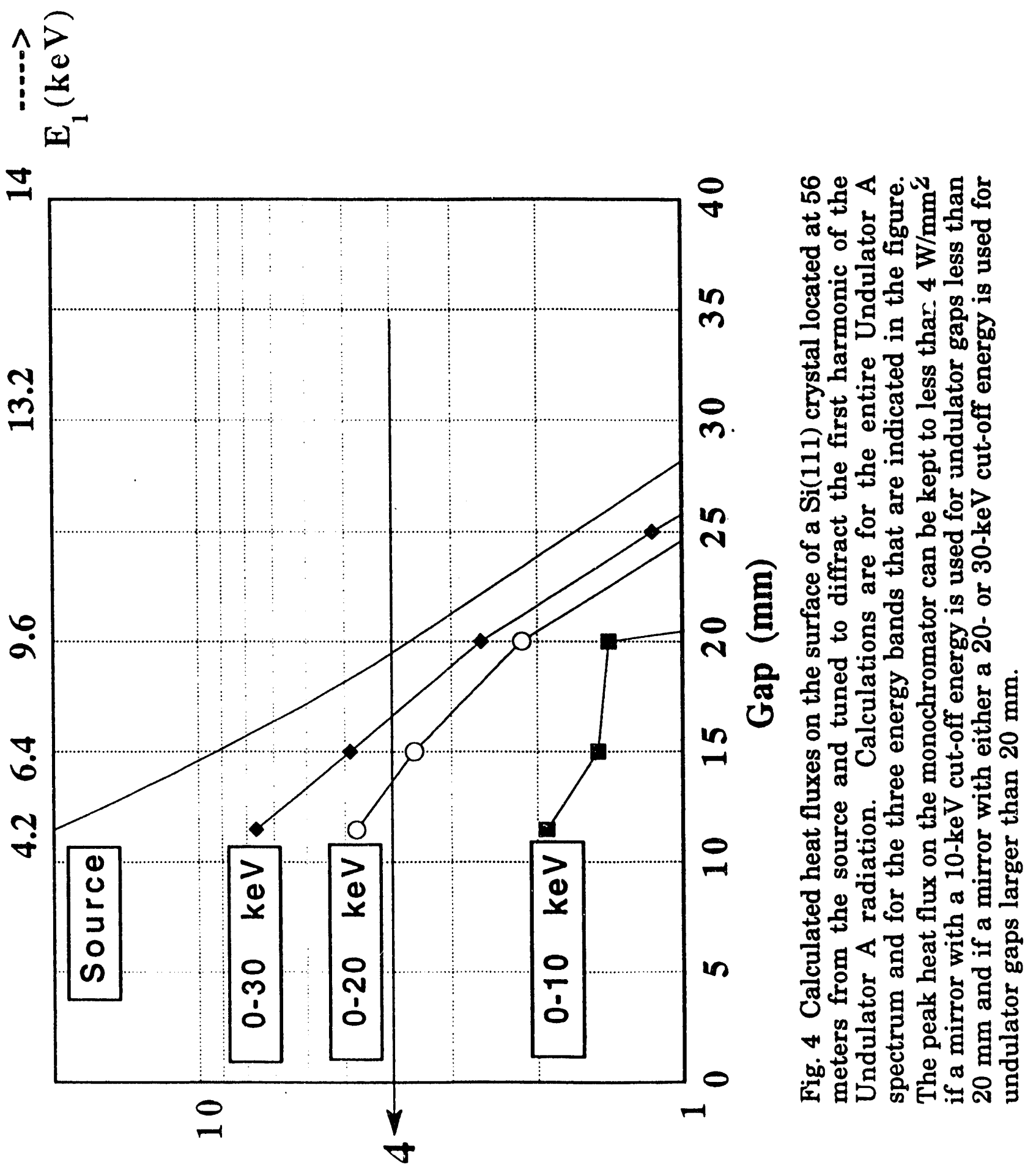

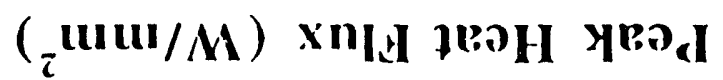




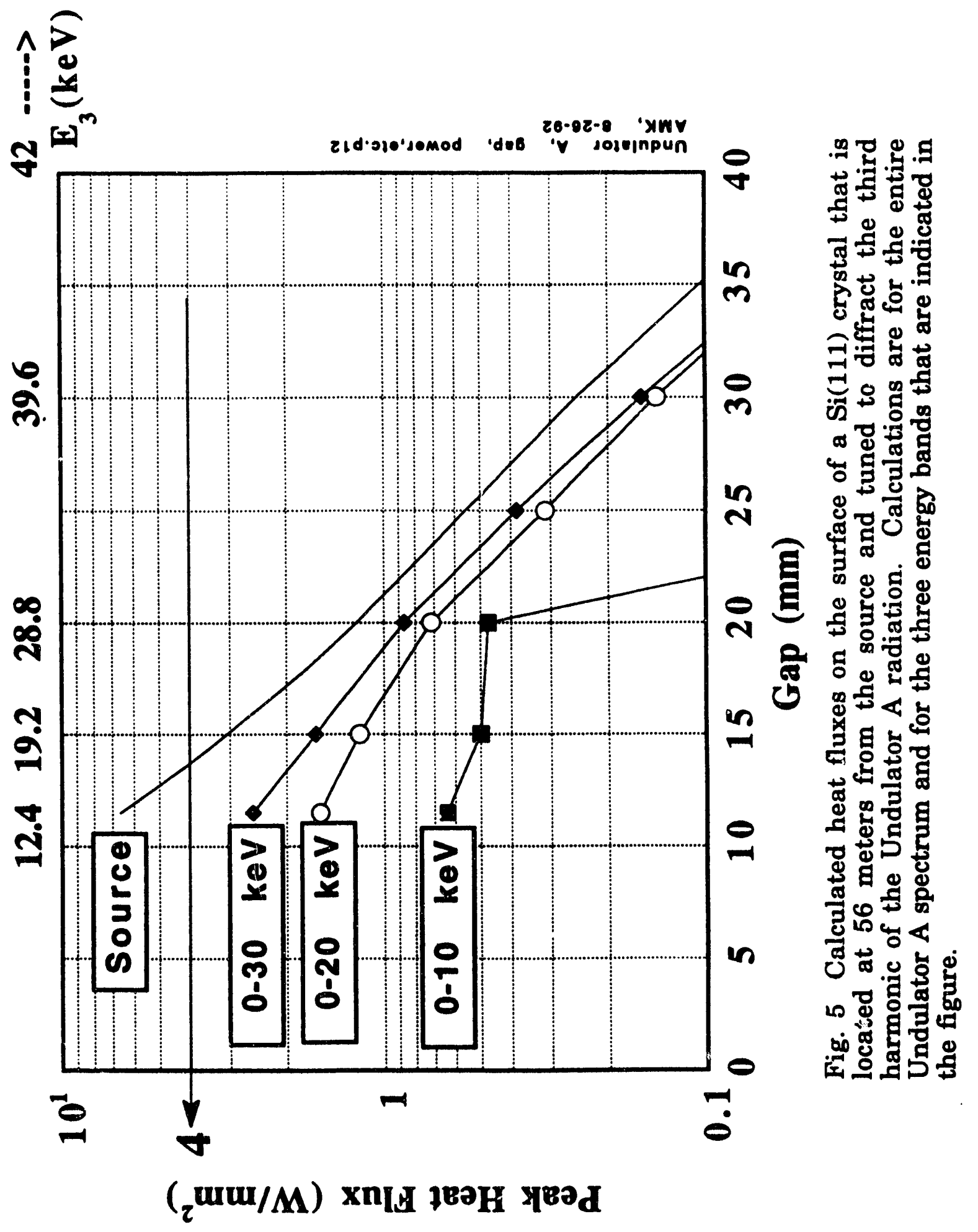




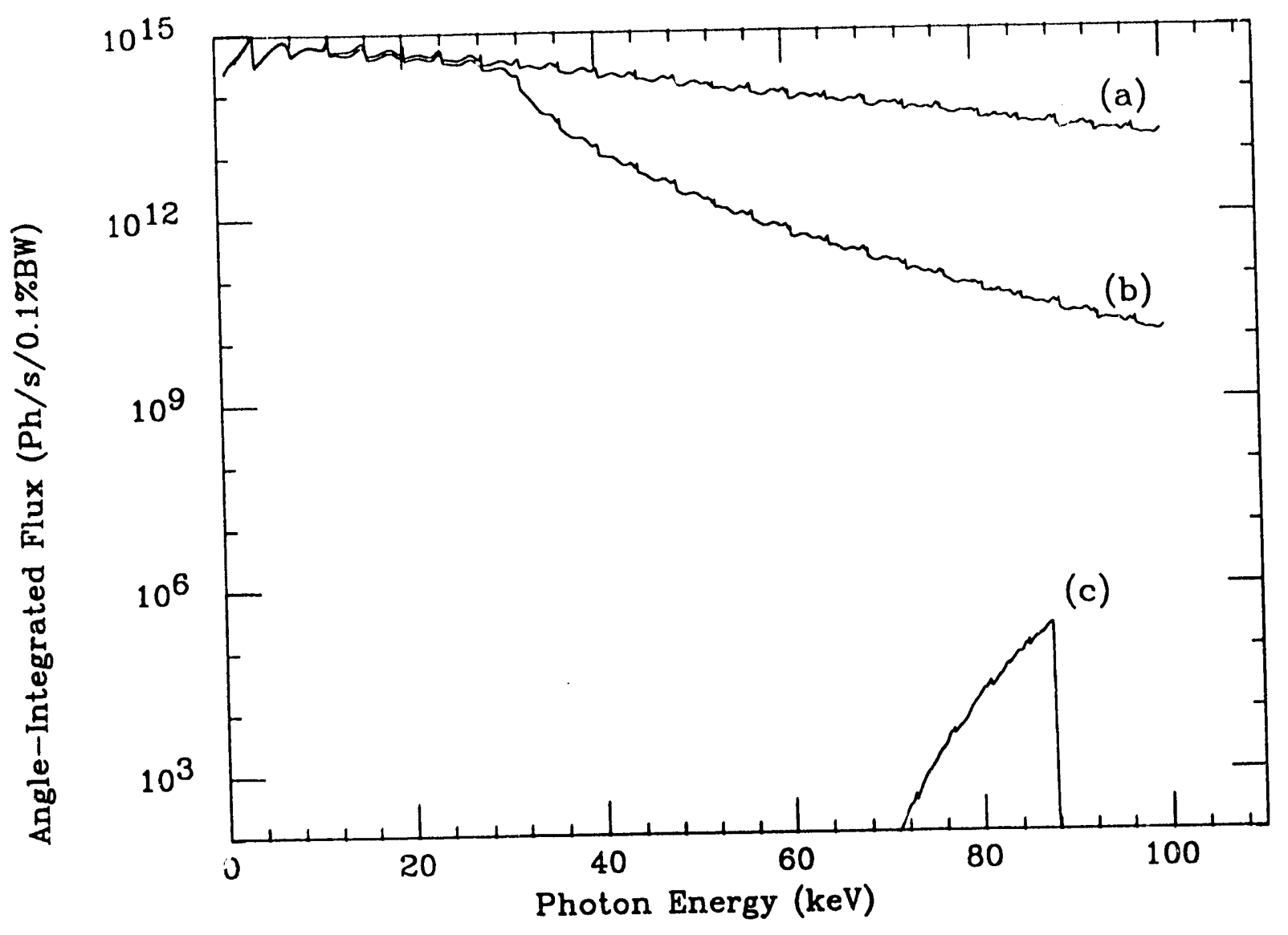

Fig. 6 Calculated spectrum of (a) the raw Undulator A spectrum at the closed gap of $11.5 \mathrm{~mm}$, (b) the same spectrum after being reflected by a $\mathrm{Pt}$ mirror at 0.15 degree of grazing incidence angle, and (c) the transmitted spectrum of $b$ through a shielding slab consisting of 0.25 inch lead and 0.125 inch of steel. 


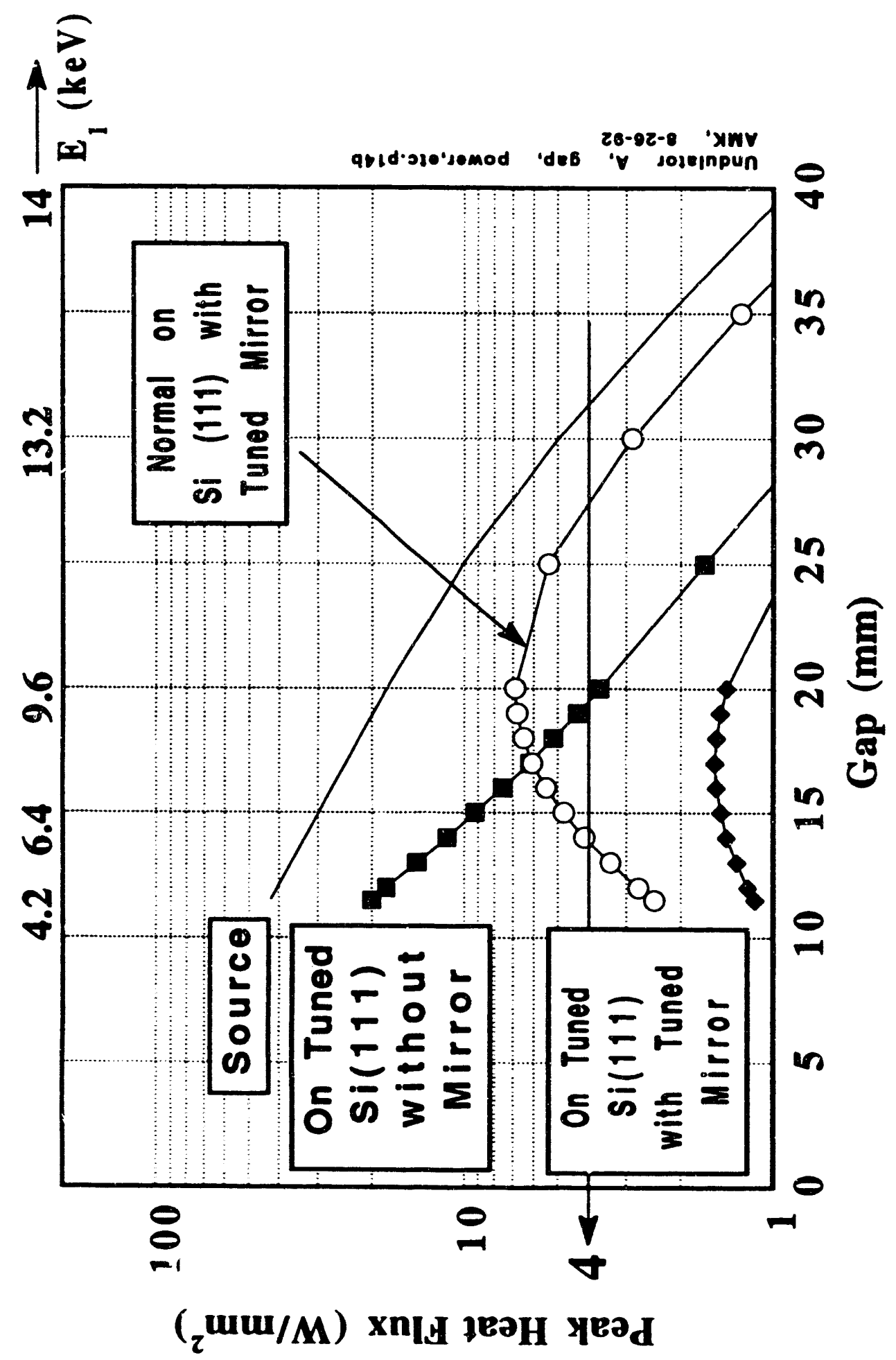

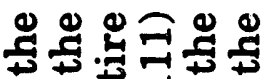

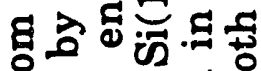
跣 언

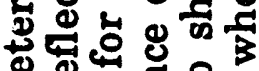
要

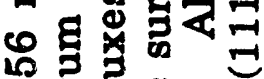
㪍造 ๘ o

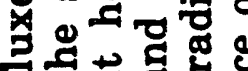
可运

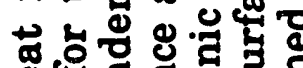
屯 s

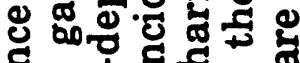
与ั

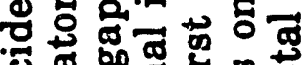

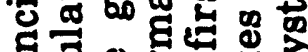

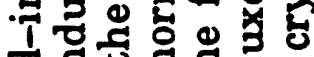
屯

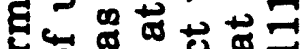

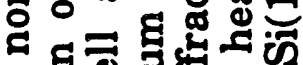
○. $\Phi$ o

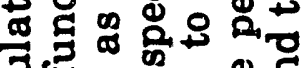
๘

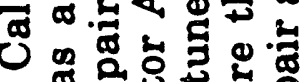

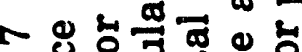
no

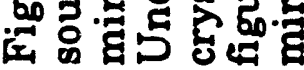



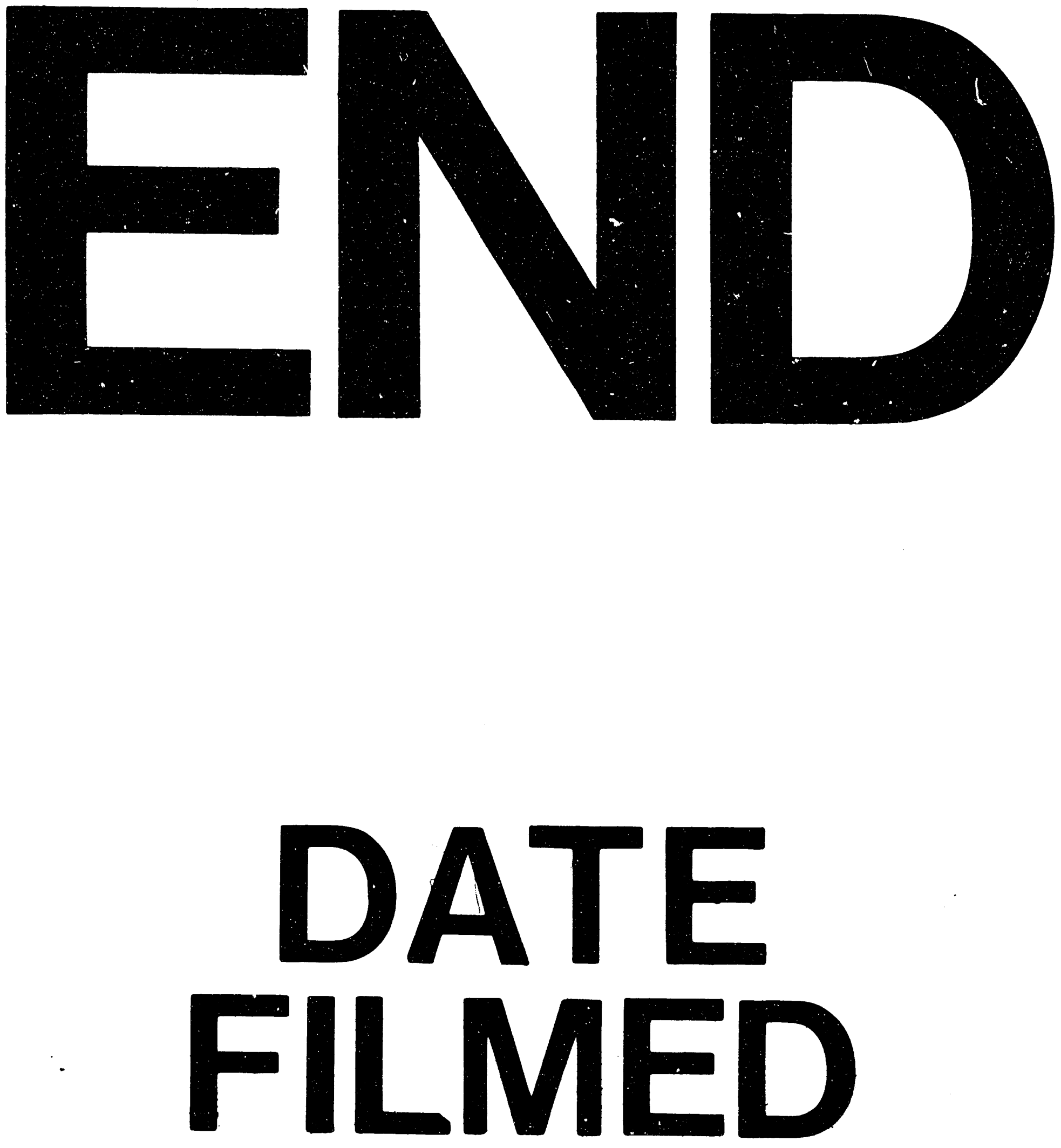

1

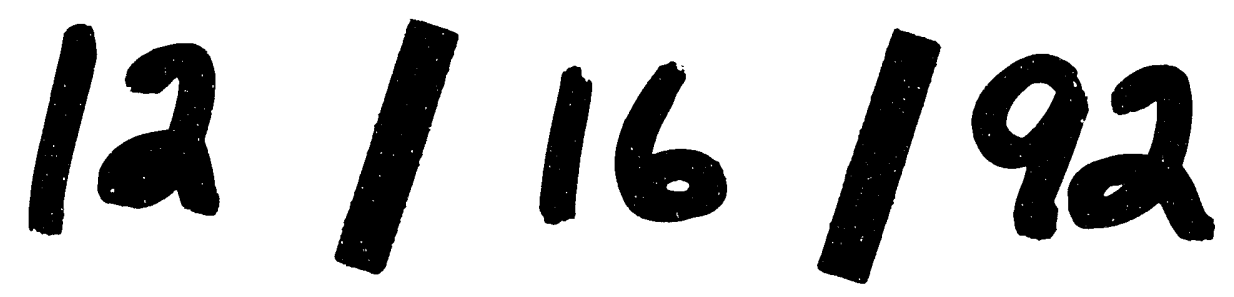


